\title{
Modeling the response of a fast ion loss detector using orbit tracing techniques in a neutral beam prompt-loss study on the DIII-D tokamak ${ }^{\text {a) }}$
}

\author{
D. C. Pace, ${ }^{1, b)}$ R. K. Fisher, ${ }^{2}$ M. García-Muñoz, ${ }^{3}$ D. S. Darrow, ${ }^{4}$ W. W. Heidbrink, ${ }^{1}$ \\ C. M. Muscatello, ${ }^{1}$ R. Nazikian, ${ }^{4}$ M. A. Van Zeeland, ${ }^{2}$ and Y. B. Zhu ${ }^{1}$ \\ ${ }^{1}$ University of California-Irvine, Irvine, California 92697, USA \\ ${ }^{2}$ General Atomics, P.O. Box 85608, San Diego, California 92186-5608, USA \\ ${ }^{3}$ Max-Planck-Institut für Plasmaphysik, Garching D-85748, Germany \\ ${ }^{4}$ Princeton Plasma Physics Laboratory, Princeton, New Jersey 08543-0451, USA
}

(Presented 17 May 2010; received 14 May 2010; accepted 28 June 2010; published online 12 October 2010)

\begin{abstract}
A numerical model describing the expected measurements of neutral beam prompt-losses by a newly commissioned fast ion loss detector (FILD) in DIII-D is presented. This model incorporates the well understood neutral beam deposition profiles from all eight DIII-D beamlines to construct a prompt-loss source distribution. The full range of detectable ion orbit phase space available to the FILD is used to calculate ion trajectories that overlap with neutral beam injection footprints. Weight functions are applied to account for the level of overlap between these detectable orbits and the spatial and velocity (pitch) properties of ionized beam neutrals. An experimental comparison is performed by firing each neutral beam individually in the presence of a ramping plasma current. Fast ion losses determined from the model are in agreement with measured losses. (C) 2010 American Institute of Physics. [doi:10.1063/1.3478996]
\end{abstract}

\section{INTRODUCTION}

The ultimate success of the tokamak as a fusion energy device depends on the ability to confine fusion products that are of considerably greater energy than the thermal background plasma. Energetic ion losses due to high-frequency $(f>50 \mathrm{kHz})$ magnetohydrodynamic modes have been inferred from bolometer arrays ${ }^{1}$ and directly observed by scintillator-based detectors. ${ }^{2}$ Losses caused by sawtooth oscillations have also been directly observed. ${ }^{3}$ The reverseorbit technique discussed here is intended to demonstrate a method by which loss measurements from a device wall can be used to infer regions of interaction (in both absolute position and wave-particle phase space) between instabilities and energetic ions. Beginning with measurements of the orbit properties of ions that strike the detector, it is possible to trace the earlier path of the ion to determine locations of overlap between it and any instability. The validity of this method rests on the assumption that the ions of interest very nearly follow trajectories prescribed by the equilibrium field during the time that elapses between the event of interest and detection at the wall. This transit time is on the order of tens of microseconds, which is much less than the slowing-down time of injected beam neutrals (tens of milliseconds).

Here, neutral beam prompt-losses are studied through a carefully designed DIII-D discharge that fires each of the eight available sources individually. Ions that are observed by the fast ion loss detector (FILD) are traced in reverse time to quantify their overlap with the injection footprint of the relevant beam source. The same framework is suggested for

\footnotetext{
a) Contributed paper, published as part of the Proceedings of the 18th Topical Conference on High-Temperature Plasma Diagnostics, Wildwood, New Jersey, May 2010.

${ }^{b)}$ Electronic mail: pacedc@ @usion.gat.com.
}

use with any particular source of fast ion emissivity. A similar approach has been previously employed ${ }^{4}$ using the trajectories of fusion products to measure the poloidal magnetic field in a tokamak. Future work will apply this procedure to the fast ion source that is generated from instabilities that drive ions onto loss orbits.

Scintillator-based energetic ion loss detectors have been used on many fusion devices since first being developed on the Tokamak Fusion Test Reactor. ${ }^{5}$ A new FILD (Ref. 6) has recently been installed for use at DIII-D. The FILD design is based on a diagnostic in use at ASDEX-U. ${ }^{7}$ These detectors use fast-response scintillators to detect light from ion impacts. A collimating aperture is placed in the heat shield surrounding the scintillator, which results in a particular mapping between the pitch angles and gyroradii of impacting ions onto the two-dimensional detector. Imaging the scintillator with a camera provides time resolved measurements of the particular ion orbits that are lost at the wall position of the detector. This information is used to trace the ion along the trajectory it followed en route to the detector.

The setup of the experimental discharge is described in Sec. II. The analysis method used to interpret FILD data and place it in context with measurements in the plasma core is discussed by way of a neutral beam prompt-loss study presented in Sec. III. A summary of the results for the example discharge is given in Sec. IV. Finally, a discussion of future applications is presented in Sec. V.

\section{NEUTRAL BEAM PROMPT-LOSS SETUP}

In order to verify the technique of reverse-orbit tracing, prompt-losses from neutral beam injection are studied in the DIII-D tokamak. Given the thorough understanding of neutral beam injection (NBI) in tokamaks (see Sec. IIB of Ref. 8 


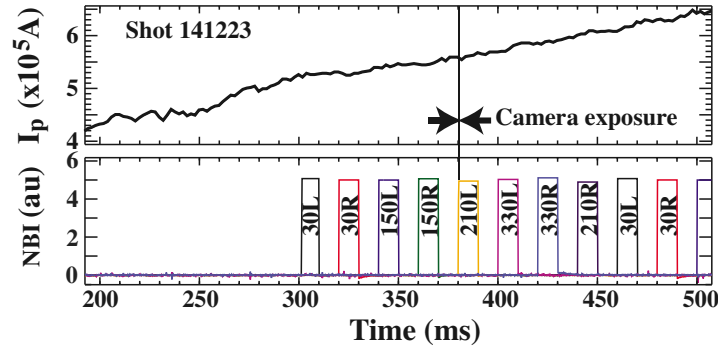

FIG. 1. (Color online) Plasma current and neutral beam injection for shot 141223. The line labeled "Camera exposure" highlights the trigger of the camera frame shown in Fig. 2. The width of this line indicates the $1 \mathrm{~ms}$ exposure time of that frame.

for an extensive reference list and Ref. 9 for more recent experimental verification of NBI theory), a DIII-D discharge was designed to inject each of its eight sources individually. With each beam pulse, a collection of prompt-losses occur. These are due to ionization events that place the energetic ion on an orbit that terminates on the vessel wall. Each neutral beam source is injected for a $10 \mathrm{~ms}$ pulse during a plasma current ramp as shown in Fig. 1 from shot 141223 with $B_{0}=2.05 \mathrm{~T}$. The purpose of ramping the plasma current is that it increases the amplitude of the poloidal magnetic field, causing variation in both the pitch angles of the energetic ions and the location at which they strike the wall. The FILD is located at the $225^{\circ}$ toroidal position in a port that is approximately $45^{\circ}$ below the midplane.

\section{FILD MODEL OF PROMPT-LOSS DETECTION}

The concept of modeling FILD measurements centers on comparing the ion orbits for which the detector is sensitive to a specific source that drives ion losses. In the case of the neutral beam prompt-loss study presented here, the source of lost ions is determined according to the injection of any particular DIII-D beamline. In the description of the method presented in this section, the specific example from a single pulse will be shown. This example is a pulse of the $210 \mathrm{~L}$ neutral beam source that occurs over $380 \leq t \leq 390 \mathrm{~ms}$. Figure 2 shows a camera image from the FILD during injection of the $210 \mathrm{~L}$ source. The camera is exposed for $1 \mathrm{~ms}$ beginning at $t=380 \mathrm{~ms}$. The frame is displayed as a negative image for clarity, meaning that the dark region appearing over

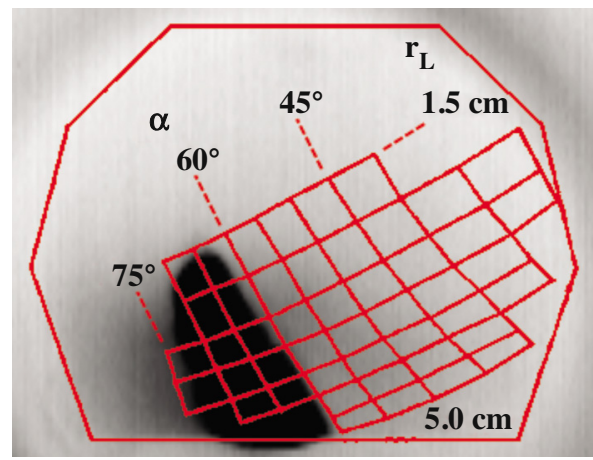

FIG. 2. (Color online) Negative image of FILD camera frame showing prompt-losses as the dark region between $60^{\circ} \leq \alpha \leq 75^{\circ}(\operatorname{shot} 141223, t$ $=380 \mathrm{~ms}$ ). pitch angles $60^{\circ} \leq \alpha \leq 75^{\circ}$ corresponds to a large flux of impacting ions. The FILD measures pitch angle $\alpha$, which is related to the commonly cited orbit pitch as $\cos (\alpha)=v_{\|} / v$, where $v_{\|}$is the particle's velocity parallel to the total magnetic field and $v$ is the particle's total velocity.

\section{A. Weight functions applied to neutral beam injection}

The objective of modeling the FILD is to accurately account for an observed signal in both amplitude and orbit phase space, given the well known source of prompt-loss ions due to neutral beam injection. The number of ions that are expected to reach the FILD $N_{\text {FILD }}$ is a function of energy $E_{0}$ and pitch $v_{\|} / v$. If the total number of ions seeded by the beam onto prompt-loss orbits is given by $N_{\mathrm{NBI}}$ $=N_{\mathrm{NBI}}\left(R, \phi, z, E_{0}, v_{\|} / v\right)$, then this must represent the largest possible signal any loss detector could observe. Any given detector is located at a fixed spatial position, however, which limits the ion orbits that can be detected to those that feature a trajectory leading to intersection with the diagnostic. These detectable orbits are accumulated, or extracted, through reducing $N_{\mathrm{NBI}}$ by applying weight functions $W$. Each weight function in this model features a value between zero and one. The number of ions expected to reach the FILD is

$$
N_{\mathrm{FILD}}\left(E_{0}, v_{\|} / v\right)=\sum_{L} W_{\text {pitch }} W_{R, \phi, z} N_{\mathrm{NBI}},
$$

where $W_{R, \phi . z}$ is the spatial weight function, $W_{\text {pitch }}$ is the pitch (or velocity) weight function, and the sum is taken over the trajectory of the ion $L$.

\section{B. Reverse-orbit trajectories}

Any measurement by the FILD provides simultaneous observations of an ion's energy, pitch, and location (knowing that the ion must have passed through the entrance aperture to reach the scintillator). Detectors of this design are restricted in the gyroradii and pitches that can be detected due to the geometry of the probe head and the collimating aperture. The range of detectable ion orbits is determined by calculating ion trajectories using the plasma's magnetic equilibrium and collecting statistics on the parameters that both reach the scintillator and avoid the probe head. The FILD at DIII-D is capable of detecting ions of gyroradius $r_{L}$ in the range $1.5 \leq r_{L} \leq 5.0 \mathrm{~cm}$ and pitch angles $\alpha$ in the range $30^{\circ} \leq \alpha \leq 75^{\circ}$. The strike map overlaid on the camera frame of Fig. 2 indicates these limits. While there are at least two well established codes for calculating these maps, neither is the subject of a detailed published treatment. The mapping shown here is calculated using EFIPDESIGN (described, but not named, in Sec. II of Ref. 10). Secondary calculations with NLSDETSIM (described, but not named, in Ref. 11) are ongoing and presently consistent with the EFIPDESIGN results.

From the total measurable orbit parameter space, a collection of ion trajectories are calculated in reverse time. These ions begin at the spatial position of the collimating FILD aperture and are traced backward in time. Orbits are calculated using the measured magnetic equilibrium and a code that solves the Lorentz force equation (a descendant of Ref. 12). Fast ion orbits are often calculated in this manner to contribute to the understanding of various diagnostic obser- 
vations (e.g., see Fig. 4 of Ref. 13 for an application with respect to fast ion $D_{\alpha}$ imaging). For the model FILD, the orbits must be calculated over a long enough time period to reproduce any overlap between the orbit and the beam footprint. The elapsed time, however, must also be short enough that the magnetic equilibrium is static and only promptlosses are captured. An optimum value is determined empirically and nearly all orbit calculations are performed within a $60 \mu$ s time window. This time period is much shorter than the tens of milliseconds that represent the slowing-down time of injected neutrals.

The FILD strike map is a function of gyroradius, which can be expressed as an energy value once the total magnetic field amplitude is known. For the model FILD that focuses on neutral beam prompt-losses, full energy surveys are not necessary. A prompt-loss ion does not thermalize because it escapes to the vessel wall too quickly. That leads to the requirement that the energy of such a detected ion must be a value that is directly seeded by the neutral beam. Due to the presence of molecular deuterium, neutral beams with a set injection energy (voltage) produce neutrals with full, onehalf, and one-third energies. Only orbit trajectories with these three energy values are calculated. For each energy value, orbits are calculated for 40 different pitch angles spaced evenly between $20^{\circ} \leq \alpha \leq 83^{\circ}$. The pitch angle range is purposely set to extend slightly beyond the FILD's detection range to ensure that any nearly detectable losses are tabulated.

\section{Spatial weighting}

The spatial weighting consists of quantifying how well a prompt-loss orbit overlaps with neutral beam injection throughout the three-dimensional space of the tokamak. With all $W_{R, \phi, z}$ initially set to zero, a value of $W_{R, \phi, z}=1$ is assigned at positions along $L$ for which the following three conditions are met.

(1) The vertical position of the ion is within $-0.5 \leq z$ $\leq 0.5 \mathrm{~m}$.

(2) The toroidal position of the ion is within the boundaries for the relevant neutral beam source.

(3) The parallel velocity of the ion is in the same direction as the injection from the neutral beam source.

Condition 1 is empirically known to encompass the entire vertical extent of any beamline. It is possible for this condition to be satisfied at a position for which there are no injected neutrals. In that situation, the injected neutral density and ionization profiles will be equal to 0 and remove any expectation of signal. Condition 2 accounts for the position of the FILD. Placing the detector at a different toroidal (or poloidal) location will affect which prompt-loss orbits are detected. While condition 3 is technically part of the pitch weighting, it is checked at this stage because it follows the same binary description as the spatial considerations (i.e., if the ion exhibits a parallel velocity that is opposite the injected value, then that orbit must be weighted to zero because it is not a valid prompt-loss candidate). The pitch weighting calculated in Sec. III E displays a more complex variation. There is no check on the major radius overlap because all injected neutral beams cover the entire major radius across their sightline.

The injection geometry of a neutral beam is described in terms of its full three-dimensional spatial extent. The toroidal extent of an injected beam is calculated from the geometry of the sightline along the beam on the vertical midplane. This is equivalent to following the toroidal coverage of the beamlines as shown in Fig. 1 of Ref. 9. The vertical extent of the neutral beams is most recently calculated and experimentally verified in Ref. 14. Accounting for both the toroidal and poloidal geometry of the FILD relative to the lost ion source provides for a rigorous model of the observations and allows for a systematic calculation of expected signals for a second FILD to be deployed on DIII-D in the future. ${ }^{15}$

\section{Prompt-loss ions seeded by neutral beams}

Determination of the number density of prompt-loss ions from neutral beam injection $N_{\mathrm{NBI}}$ is achieved by calculating ionization profiles based on modeled neutral density profiles. These densities are determined according to the procedure developed in Ref. 14, which applies a standard "pencil beam" treatment repeatedly to construct a density profile across the $R z$-plane. From the injected neutral density profiles, the ionization profile is calculated by taking the gradient along the major radius for each value of constant $z$ because ionization is due to electron impacts and charge exchange events. This sets the value of $N_{\mathrm{NBI}}$ as a function of position. Beam injection paths and energies are known, along with the magnetic equilibrium, and since each position corresponds to a primary injected pitch angle as well, we have $N_{\mathrm{NBI}}=N_{\mathrm{NBI}}\left(R, \phi, z, E_{0}, v_{\|} / v\right)$.

The numerical value of $N_{\mathrm{NBI}}$ is meaningful, though it is treated as arbitrary in this study and only relative differences as a function of ion energy and pitch are considered. Promptloss signals in DIII-D are typically large enough to saturate the charge-coupled device camera of the FILD, making calibrated lost ion fluxes impossible to determine. Improving this technique and the measurements (partially through an array of photomultiplier tubes that record the signal from narrow regions of the scintillator with high time resolution and no saturation) to quantitatively verify detected lost ion flux levels is a future objective.

\section{E. Pitch weighting}

For every ion path, at every position for which $W_{R, \phi, z}$ $\neq 0$, the pitch weight factor $W_{\text {pitch }}$ is calculated. The spatial weight is calculated first for the computational benefit of reducing the number of pitch operations that need to be performed (i.e., there is no reason to attempt a pitch weight calculation at a position where there are no injected neutrals). Pitch angles seeded by a neutral beam along the major radius are calculated as the angle between this sightline of the neutral beam and the toroidal magnetic field using $v_{\|}=v_{\phi}$. This is considered the ideal injected pitch angle.

It is necessary to describe the entire range of injected pitch angles in order to determine whether a prompt-loss ion trajectory is capable of originating from any given beam 


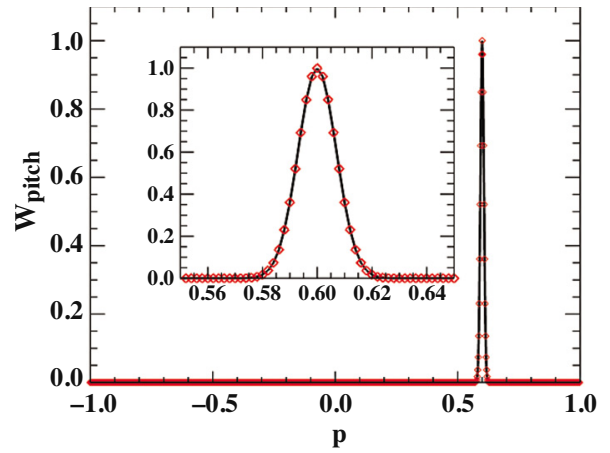

FIG. 3. (Color online) Pitch weight function for $p_{\mathrm{NBI}}=0.6$. The red diamonds indicate the discrete points as encountered in the model.

source. The modeled variation about this ideal value is treated by way of a Gaussian spread defined according to horizontal ( $R \phi$-plane) divergence of the beam. Horizontal divergence is used because the ideal pitch is defined using only the toroidal field, making only the horizontal divergence relevant. The horizontal divergence $\theta_{h}=8.73 \times 10^{-3}(\mathrm{rad})$ is smaller than the vertical ( $R z$-plane) divergence $\theta_{v}=2.27$ $\times 10^{-2}$ (rad), resulting in a stronger constraint (Fig. 3 provides an example of the narrow region that defines an overlap in pitch). These divergences have been measured or tested in experimental work ${ }^{14}$ and are used in both TRANSP (Ref. 16) modeling and fast ion $D_{\alpha}$ spectroscopy simulations ${ }^{17}$ for each of DIII-D's neutral beams. At every position where a pitch weight is calculated, this value is described as a Gaussian of unity amplitude centered on the ideal value with a width (standard deviation, $\sigma_{p}$ ) equal to the horizontal divergence of the neutral beam. The pitch weight term is described in functional form according to

$$
\begin{aligned}
& p_{\text {ion }}=v_{\|} / v, \\
& \sigma_{p}=p_{\mathrm{NBI}}-\cos \left[\cos ^{-1}\left(p_{\mathrm{NBI}}\right)+\theta_{h}\right], \\
& W_{\text {pitch }}=\exp \left[-\frac{1}{2}\left(\frac{p_{\text {ion }}-p_{\mathrm{NBI}}}{\sigma_{p}}\right)^{2}\right],
\end{aligned}
$$

where $p_{\mathrm{NBI}}=p_{\mathrm{NBI}}(R, \phi, z)$ is the ideal injected pitch angle of the neutral beam.

An example of $W_{\text {pitch }}$ is shown in Fig. 3, which sets $p_{\mathrm{NBI}}=0.6$ (resulting in $\sigma_{p}=0.007$ ). In the model, as in the figure, the pitch axis is divided into 1000 elements covering $-1.0 \leq v_{\|} / v \leq 1.0$. Figure 3 demonstrates the narrow range of pitches expected from neutral beam injection. The value of $W_{\text {pitch }}$ is determined from this type of profile using an interpolation, therefore, the resolution as shown in the inset of Fig. 3 is sufficient.

\section{F. Expected FILD observations}

At this point, it should be clarified that the technique described here focuses on proof-of-principle demonstration and is not capable of estimating absolute flux levels. The units of $N_{\mathrm{NBI}}$ are not those of emissivity (number/volume/ time) because it is difficult to define the proper path length given the three-dimensional geometry of neutral beams. The next iteration of this model will treat the production of prompt-loss ions as a true emissivity, with the proper units,

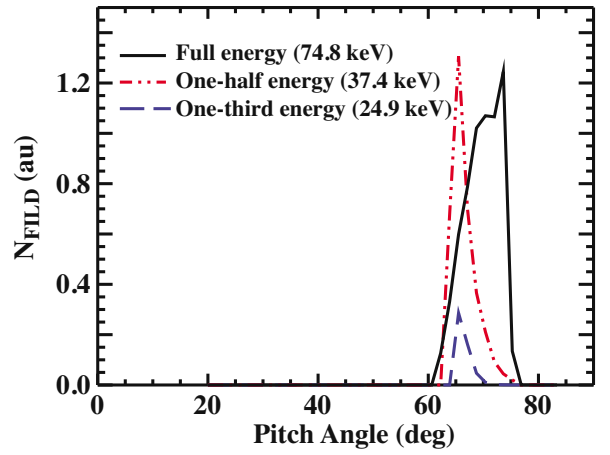

FIG. 4. (Color online) Expected FILD observation density for the 210L example case presented throughout this description.

thereby allowing for absolute flux level calculations. The present results are in arbitrary units and are useful to study the observed pitch angle ranges of prompt-losses. The results from the $210 \mathrm{~L}$ case are presented in Fig. 4 . With a magnetic field at the FILD of $B_{\mathrm{FILD}}=1.51 \mathrm{~T}$, the corresponding gyroradii for the full, one-half, and one-third energies are approximately $3.8,2.7$, and $2.2 \mathrm{~cm}$, respectively. Figure 4 contains the same information as the FILD camera data in Fig. 2. Qualitative comparisons are excellent as they both indicate losses featuring a $60^{\circ} \leq \alpha \leq 75^{\circ}$ pitch angle range and all injection energies. The upper limit at $75^{\circ}$ must be tempered by the fact that it is also the edge of the detectable range for the FILD. This upper limit of detectable pitch angle also makes it difficult to confirm the narrower pitch angle ranges at lower energy, which are shown in the model result of Fig. 4.

The model results support the continuous band seen in the camera data because the gyroradius resolution of the FILD is on the order of $1 \mathrm{~cm}$. Resolution is a function of gyroradius and pitch angle, however, and it must be calculated using a code such as EFIPDESIGN or NLSDETSIM for each gyroradius/pitch angle pair. This fully determined resolution will be convolved with the model results to generate expected FILD camera frames.

\section{SURVEY OF SHOT 141223}

A comparison between the model, or expected, pitch angle observations and the FILD camera data is presented in Fig. 5. Each neutral beam source is depicted by a unique symbol with black representing experimental observations and red/gray illustrating the model results. The plasma current is evolving during each $10 \mathrm{~ms}$ neutral beam pulse; the range covered is narrower than the width of the symbols used in the plot. Vertical error bars represent the entire range of pitch angles for which either the camera data or the model returned losses. Some error bars and plotting symbols in the range above the $75^{\circ}$ pitch angle have been omitted for clarity. For the FILD camera data, the pitch angle error bars also add $\pm 5^{\circ}$ to represent the uncertainty in pitch angle measurement that is caused by the vibration of the optics platform to which the camera is attached. The blue horizontal lines indicate the boundary of pitch angles that the FILD is capable of detecting. Model data well outside of this range should not be detected by the FILD, though values very close to the 


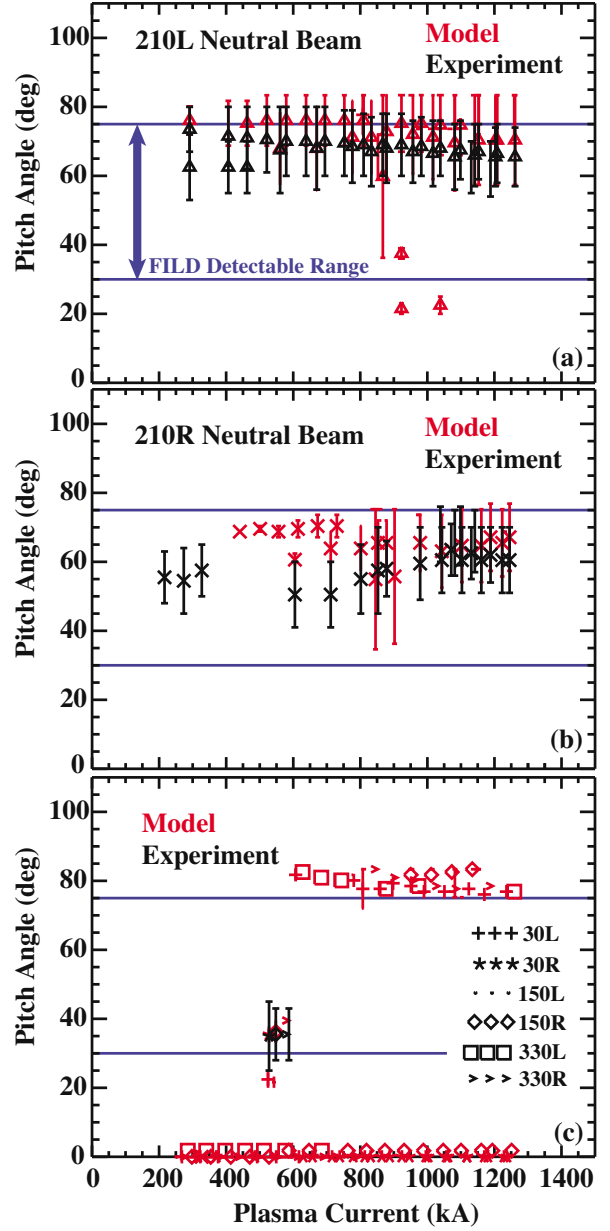

FIG. 5. (Color online) Pitch angle of ion losses at full beam energy as measured (black) and as calculated from the model (red/gray) for the (a) 210L, (b) 210R, and (c) all other neutral beam sources.

boundary may be observed due to FILD resolution. Cases for which there is no observed loss in the FILD camera data are not placed in the plot because they would obscure the zero result from the model (null results from the model are displayed as less than $5^{\circ}$ pitch angle).

Figure 5 indicates that the model developed here to describe neutral beam prompt-loss detection by the DIII-D FILD is capable of accurately reproducing the experimentally observed results. The 210L neutral beam, denoted by the $\Delta$ symbol, is shown in the top panel (a) of Fig. 5 and is well described at all values of plasma current. Model results for the $210 \mathrm{~L}$ source occurring below $40^{\circ}$ pitch angles may be due to numerical error. The $210 \mathrm{R}$ beam $(x)$, shown in the middle panel (b) of Fig. 5, is well described at the larger values of plasma current and further study is required to uncover the cause of discrepancies for $I_{p}<800 \mathrm{kA}$. Few prompt-loss results are observed in the FILD camera data for neutral beams located at other toroidal positions; however, the observations for the $30 \mathrm{R}, 150 \mathrm{R}$, and $330 \mathrm{R}$ sources near $500 \mathrm{kA}$ are reproduced by the model and shown in the bottom panel (c) of Fig. 5. All neutral beams produce promptloss populations, so the lack of an observed signal by the
FILD is due to either its wall position (i.e., the prompt-losses strike at other toroidal and poloidal positions) or its detection range (i.e., the prompt-losses occur at pitch angles beyond the detection range shown in Fig. 4).

\section{FUTURE APPLICATIONS}

The study presented here focuses on the well established physics of neutral beam injection into a tokamak for the purpose of validating this conceptual treatment. Future application of this technique is aimed at improving understanding of fast ion losses driven by Alfvén eigenmodes (AEs). For this more complicated study, it will be necessary to calculate the lost ion source function using a code that accounts for interactions between fast ions and AEs. It is suggested that such a code, ORBIT, ${ }^{18}$ could provide the source for the model FILD by following perturbed ion orbits to the last closed flux surface. The distribution of these ions along the last closed flux surface is analogous to the prompt-loss ion density of the neutral beam case. Such an effort will lead to a quantitative comparison between expected and measured fast ion losses, thereby providing valuable constraining data from which to improve the relevant transport models.

\section{ACKNOWLEDGMENTS}

This work supported in part by the U.S. DOE under Grant Nos. SC-G903402, DE-AC02-09CH11466, and DEFC02-04ER54698.

${ }^{1}$ H. H. Duong, W. W. Heidbrink, E. J. Strait, T. W. Petrie, R. Lee, R. A. Moyer, and J. G. Watkins, Nucl. Fusion 33, 749 (1993).

${ }^{2}$ M. García-Muñoz, H.-U. Fahrbach, S. Günter, V. Igochine, M. J. Mantsinen, M. Maraschek, P. Martin, P. Piovesan, K. Sassenberg, and H. Zohm, Phys. Rev. Lett. 100, 055005 (2008).

${ }^{3}$ Y. I. Kolesnichenko, V. V. Lutsenko, R. B. White, Y. V. Yakovenko, and S. J. Zweben, Phys. Plasmas 6, 1117 (1999).

${ }^{4}$ W. W. Heidbrink and J. D. Strachan, Rev. Sci. Instrum. 56, 501 (1985).

${ }^{5}$ S. J. Zweben, Nucl. Fusion 29, 825 (1989).

${ }^{6}$ R. K. Fisher, D. C. Pace, M. García-Muñoz, W. W. Heidbrink, C. M. Muscatello, M. A. Van Zeeland, and Y. B. Zhu, Rev. Sci. Instrum. 81, 10D307 (2010).

${ }^{7}$ M. García-Muñoz, "Fast response scintillator based detector for MHD induced energetic ion losses in ASDEX Upgrade," Ph.D. thesis, LudwigMaximillian-University of Munich, 2006.

${ }^{8}$ W. W. Heidbrink and G. J. Sadler, Nucl. Fusion 34, 535 (1994).

${ }^{9}$ W. W. Heidbrink, M. Murakami, J. M. Park, C. C. Petty, M. A. Van Zeeland, J. H. Yu, and G. R. McKee, Plasma Phys. Controlled Fusion 51, $125001(2009)$

${ }^{10}$ A. Werner, A. Weller, and D. S. Darrow, Rev. Sci. Instrum. 72, 780 (2001).

${ }^{11}$ S. J. Zweben, R. L. Boivin, M. Diesso, S. Hayes, H. W. Hendel, H. Park, and J. D. Strachan, Nucl. Fusion 30, 1551 (1990).

${ }^{12}$ J. Felt, C. W. Barnes, R. E. Chrien, S. A. Cohen, W. W. Heidbrink, D. Manos, and S. Zweben, Rev. Sci. Instrum. 61, 3262 (1990).

${ }^{13}$ M. A. Van Zeeland, W. W. Heidbrink, and J. H. Yu, Plasma Phys. Controlled Fusion 51, 055001 (2009).

${ }^{14}$ M. A. Van Zeeland, J. H. Yu, N. H. Brooks, W. W. Heidbrink, K. H. Burrell, R. J. Groebner, A. W. Hyatt, T. C. Luce, N. Pablant, W. M. Solomon, and M. R. Wade, Plasma Phys. Controlled Fusion 52, 045006 (2010).

${ }^{15}$ R. K. Fisher and W. W. Heidbrink (private communication, 2010).

${ }^{16}$ See http://w3.pppl.gov/transp/ for information on TRANSP and its uses.

${ }^{17}$ W. W. Heidbrink, K. H. Burrell, Y. Luo, N. A. Pablant, and E. Ruskov, Plasma Phys. Controlled Fusion 46, 1855 (2004).

${ }^{18}$ R. B. White and M. S. Chance, Phys. Fluids 27, 2455 (1984). 